\title{
Musique de métissage pan-balkanique en Roumanie
}

Speranța Rădulescu

\section{(2) OpenEdition}

Journals

Édition électronique

URL : http://journals.openedition.org/ethnomusicologie/701

ISSN : 2235-7688

Éditeur

ADEM - Ateliers d'ethnomusicologie

Édition imprimée

Date de publication : 1 janvier 2001

Pagination : 151-162

ISBN : 2-8257-0723-6

ISSN : $1662-372 X$

\section{Référence électronique}

Speranța Rădulescu, « Musique de métissage pan-balkanique en Roumanie », Cahiers

d'ethnomusicologie [En ligne], 13 | 2001, mis en ligne le 09 janvier 2012, consulté le 19 avril 2019. URL : http://journals.openedition.org/ethnomusicologie/701

Ce document a été généré automatiquement le 19 avril 2019.

Tous droits réservés 


\title{
Musique de métissage pan- balkanique en Roumanie
}

\author{
Speranța Rădulescu
}

\section{NOTE DE L'ÉDITEUR}

Ce texte a été présenté sous une forme légèrement abrégée lors du colloque international « La diffusion des musiques du monde », organisé à Paris du 15 au 17 mai 1998 par Zone Franche, l'Institut du Monde Arabe et l'Université de Bourgogne.

1 Dans les années 1970, pendant les fêtes populaires du Sud de la Roumanie, on entend une musique d'ensemble (taraf) d'un style plutôt incertain, qui joue dans un espace sonore délimité par trois repères : la musique de faubourgs de Valachie (province située entre le cours inferieur du Danube et la branche meridionale des Carpates), la musique rurale et urbaine du Banat (province jouxtant la frontière serbo-roumaine) et la «novocomponovana narodna muzika » de Yougoslavie (Broughton et al. 1994 : 83). Cette nouvelle musique est interprétée par des taraf à composition renouvelée, comprenant, grosso modo, des trompettes, des taragot $^{1}$, des saxophones, des synthétiseurs et des instruments de percussion, le tout amplifié par des sonorisations d'habitude minables. Cette musique semble être le fruit d'une modernisation un peu déviante et barbare des musiques populaires rurales et urbaines. Les uns l'appellent «musique du Banat», les autres «musique serbe».

\section{Une musique à identité ambiguë}

2 Vers les années 1980, la dite musique «du Banat» ou "serbe » s'éloigne de toutes les musiques existantes ou connues. La plupart des Roumains la considèrent, et la nomment déjà avec conviction, soit « serbe », soit « tsigane », en indiquant par ce fait qu'elle n'est pas tout à fait «la leur». Pourtant les musiciens professionnels d'un certain âge sont d'avis qu'elle est, en réalité, une « musique de lăutar ${ }^{2}$ » (muzică lăutărească) : ce qui signifie 
qu'ils ne s'empressent pas de l'attribuer à l'ethnie tsigane dont ils font souvent partie, mais préfèrent lui trouver une origine du côté de la corporation professionnelle des lăutar , qui est en majorité tsigane, mais pas totalement. Cette musique connaît un succès apparemment extraordinaire; elle se répand rapidement vers le nord et vers l'est, commençant même à pénétrer les régions roumaines les plus conservatrices: la Transylvanie et la Bucovine. Les Roumains croient qu'elle a été «importée » du Banat serbe $^{3}$ par les lăutar qui franchissent souvent la frontière pour jouer aux fêtes de noces de leurs voisins. Ceux qui s'y rendent fréquemment confirment qu'en Yougoslavie on joue et on chante "la même musique ", mais avec des vers en langue serbo-croate. Les Roumains, qui se déplacent rarement à l'étranger, estiment que la nouvelle musique ne peut leur venir que de là-bas - car il va de soi qu'elle doit nécessairement «venir » ou " être importée » de quelque part, tout comme un objet physique. Pourtant, les voyageurs les plus assidus remarquent avec étonnement que les Serbes croient cette musique originaire du Banat roumain! Avec ses rythmes rappelant ceux de la pop et du rock, elle est considérée par les jeunes habitués de musique légère et de musique disco comme moderne, urbaine et prestigieuse. Dans les fêtes de noces de certaines régions campagnardes, la musique banato-serbo-tsigane remplace la « musique légère ", celle qui, normalement, coexiste depuis trois décennies - en position d'infériorité - avec la musique traditionnelle.

Fig. 1 : Musiciens de Bucarest.

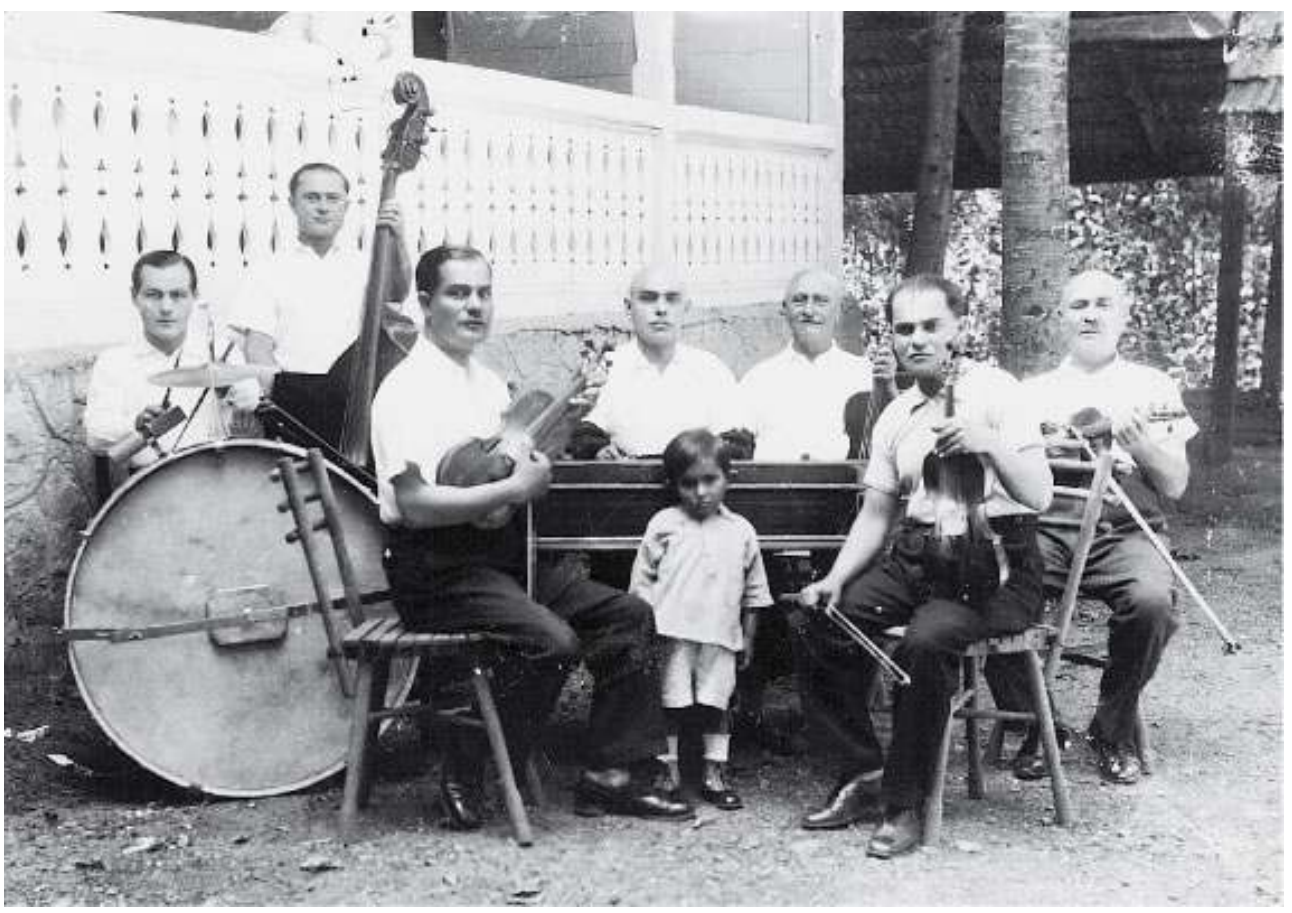

Photo : Archives du Musée du paysan roumain, vers 1930.

3 La musique « serbe » ou « du Banat » - peu importe l'étiquette - n'est jamais gravée sur disques ni diffusée à la radio et à la télévision ${ }^{4}$ : conformément aux exigences politiques, les médias roumains doivent se consacrer exclusivement au "folklore» officiel. Les personnes qui gravitent autour du pouvoir disent même qu' « elle est mal vue ». Pour leur part, les intellectuels la considèrent odieuse et n'y prêtent pas attention. (A l'exception de 
ses élites, la classe intellectuelle roumaine a toujours été plutôt indifferente à la culture populaire.)

\section{Une musique « politiquement incorrecte »}

4 Après 1980, la mode de la musique banato-serbo-tsigane bat son plein. A Timisoara (la principale ville du Banat roumain) et alentour surgissent des studios pirates, aménagés dans des caves secrètes, où des musiciens-lăutar (surtout de ceux qui entretiennent des rapports troubles avec les autorités) l'enregistrent alors que des promoteurs clandestins se chargent de la multiplier de façon rudimentaire pour la distribuer sur le marché noir à des prix « exorbitants » (l'equivalent approximatif de 15 à 20 US\$ la cassette). Depuis que les cassettophones sont devenus plus accessibles au commun des mortels, cette musique jaillit de partout: les chauffeurs la diffusent dans leur bus, les petits commercants au marché, les paysans les jours de fête sur leur véranda, les citadins dans leur appartement HLM (pour le bonheur ou le malheur des voisins) et les jeunes gens l'« emportent » dans leurs promenades dominicales. Les samedis soirs et les dimanches, elle est pratiquement inévitable, tant elle surgit et vous agresse de presque partout. La plupart du temps, cette musique partage avec la musique folklorique officielle le rôle de musique d'ambiance.

$5 \mathrm{Au}$ moment où la popularité de cette nouvelle musique prend des proportions significatives, les dirigeants communistes de l'époque réagissent. Les idéologues et les activistes du parti, censés "résoudre ce problème », sont alarmés pour deux raisons : 1 . créée sans leur accord ni leur bénédiction, cette musique s'avère incontrôlable, donc dangereuse; 2. qu'elle soit serbe, tsigane ou des faubourgs, elle est en tout cas «étrangère » et, en tant que telle, porte atteinte à la «spécificité nationale »: il s'agit, naturellement, d'une fausse spécificité, forcément paysanne, construite et promue par les médias, que tout Roumain est censé défendre (sous-entendu: du défi de ceux qui la mettraient éventuellement en doute) et dont il doit être fier. Sur ordre des chefs du parti unique, la presse déclenche une virulente "campagne de décontamination du folklore " soutenue par une bonne partie des spécialistes (ethnomusicologues et folkloristes). La campagne est suivie de "mesures " administratives dirigées contre les interprètes qui colportent «la musique polluée». La milice, qui est autorisée à surveiller leurs performances publiques, a le devoir de leur imposer des amendes, ou même de suspendre leur permis de libre pratique du métier. On ignore comment «la milice » peut décider si la musique est purement roumaine, "serbe ", "tsigane », ou "polluée » c'est-à-dire politiquement incorrecte. Mais peu importe : aucun lăutar n'est puni, car des pots de vin bien placés aident les serviteurs du pouvoir à comprendre « comme il faut» la musique de noces.

\section{Une musique qui fait irruption}

Après 1989, tous les interdits sont levés d'un coup. Les maisons d'édition privées, à peine constituées, se pressent d'enregistrer la nouvelle musique sur des cassettes et d'en saturer le marché. La musique est d'autant plus appréciée qu'elle avait été pourchassée auparavant ${ }^{5}$. Dès que la tentation du « fruit défendu » s'évanouit et que le pouvoir d'achat de la population diminue, la production et la consommation baissent, mais elles restent toujours élevées. Dorénavant, les organisateurs des fêtes populaires engagent des 
musiciens capables de la produire. Pour faire face à cette exigence, les jeunes lăutar apprennent à toute vitesse à jouer de nouveaux instruments et répertoires. Les vieux, incapables de se recycler, se sentent durement menacés par leurs cadets - surtout par les Roumains, nouveaux-venus dans le métier. Le taraf de noces, ensemble musical populaire, change au point de devenir méconnaissable: il conserve éventuellement son ancien noyau instrumental (constitué, selon les régions, de violons, de cymbalums, d'altos, d'accordéons et de contrebasses), mais transforme tout le reste en section mobile, dans laquelle les instruments "classiques" sont ou peuvent être remplacés par les instruments au goût du jour. Dans sa formule « conservatrice ", le taraf continue d'assurer la musique strictement rituelle et celle des cortèges ; dans sa formule "modernisée ", il exécute, pendant les grands repas nuptiaux, des pièces du répertoire traditionnel, mais surtout de la musique en vogue. Au Nord de la Roumanie, dans le pays de l'Oach, le taraf traditionnel local reste intouché, mais il se voit doublé d'un ensemble soit disant " urbain », les deux formations jouant alternativement la musique du pays et la muzică domnească ou «musique des Messieurs », appellation suggérant que, avant de regagner la campagne, celle-ci a fait un «détour » par la ville, pour se rafraîchir et se mettre en accord avec la vie contemporaine.

\section{Une musique tsigane?}

7 Une partie des lăutar de tout âge, mais surtout les vieux, se sentent oppressés par la nouvelle musique. Les efforts consentis pour s'y accomoder les fatiguent, dans la mesure où elle s'écarte sensiblement de la musique qui leur est familière et où ses transformations se succèdent à un rythme vertigineux, presque insoutenable. Juste après 1989, elle traverse une période "turque ": les commerçants roumains découvrent en Turquie (pays béni qui n'exige pas de visa d'entrée et qui est donc fort visité par les Roumains!) une musique semblable, qu'ils "emmènent" à la maison sous forme d'échantillons-cassettes et qu'ils font imiter par les musiciens autochtones qui la remodèlent en effaçant l'empreinte turque - d'ailleurs pâle, sinon illusoire. Au bout de quelque temps, les gens se rendent compte une fois de plus qu'ils ne savent pas comment la dénommer. Leur confusion s'accroît à mesure que certains d'entre eux multiplient leurs voyages dans les Balkans et remarquent qu'en Bulgarie, en Grèce et dans tous les pays de l'ex-Yougoslavie on retrouve des musiques très apparentées à leur musique « du Banat $» / \ll$ serbe $» /$ «turque $»^{6}$. Les Roumains utilisent encore ces qualificatifs, mais ils commencent à s'orienter avec plus de confiance vers celui de "tsigane ", en évitant ainsi toute précision concernant ses origines : les Tsiganes viennent de partout et de nulle part, ils sont présents partout dans les Balkans, ils sont partout les musiciens des "autres». Une partie des dirigeants Rom «empereurs» (sic), « rois» (sic), boulibaches, chefs de partis politiques ou tout simplement leaders d'opinion incitent leurs sujets ou leurs membres à se l'approprier et à la promouvoir par le truchement des ensembles folkloriques tsiganes. Leur propagande - ou peut-être les changements "naturels" survenus dans un climat idéologique rafraîchi - porte ses fruits : les Tsiganes s'emparent résolumment de la nouvelle musique, qui leur correspond suffisamment pour qu'ils essaient d'en faire une musique emblématique de leur ethnie. Parfois, au cours de leurs fêtes, ils ne chantent, ne jouent ni n'écoutent rien d'autre. Cette musique paraît être devenue proprement «tsigane ». Mais les lăutar âgés, qu'ils soient roumains ou tsiganes, s'entêtent à l'appeler muzică $l$ ăutărească. De leur côté, quelques dirigeants Rom 
commencent à exprimer des réserves à son sujet. Ce qui les inquiète le plus, c'est son éclectisme un peu trop frappant, trop dépersonnalisant. Quelques jours avant que j'écrive ces lignes, j'ai entendu à la télévision un leader rom, fort autorisé en matière musicale (fils d'un violoniste "classique ", lui-même violoniste), déplorant les accents orientaux qui mettent en danger la pureté et la spécificité de la vraie musique tsigane. Il s'agit d'un fait «normal» dans la société post-communiste de Roumanie: les victimes de la purification ethnique de l'ancien régime aspirent à leur tour à la " purification ethnique " de leur musique - une musique qui, à vrai dire, est à peine en train de se modeler. L'attitude du chef tsigane est partiellement due à son éducation, forcément redevable à l'idéologie nationaliste-communiste ; mais, à mon avis, elle a aussi d'autres explications : elle indique d'une part que les rapports de cette musique avec l'ethnie tsigane ne sont pas tout à fait dépourvus d'équivoque; d'autre part, elle reflète bien le désir justifié des Tsiganes de ne pas devenir les victimes d'une discrimination positive, leur imposant de revendiquer des biens qu'ils ne sont pas encore prêts à assumer.

Il y a six ans, en Suisse, un autre leader tsigane de Roumanie, sociologue instruit, intelligent et large d'esprit, déplorait le fait que les medias occidentaux, dans leur enthousiasme pour les Rom récemment redécouverts et hautement valorisés, ont tendance à faire passer n'importe quelle musique roumaine pour de la musique tsigane, ce qui n'est pas seulement faux, mais aussi un peu désagréable, tant pour les Tsiganes que pour les Roumains. (Il se référait surtout à la musique du fameux ensemble «Taraf de Haïdouk », qui s'est vue attribuer le qualificatif «tsigane » dont elle ne pourra jamais se débarrasser.)

Fig. 2 : Musiciens tsiganes du Banat.

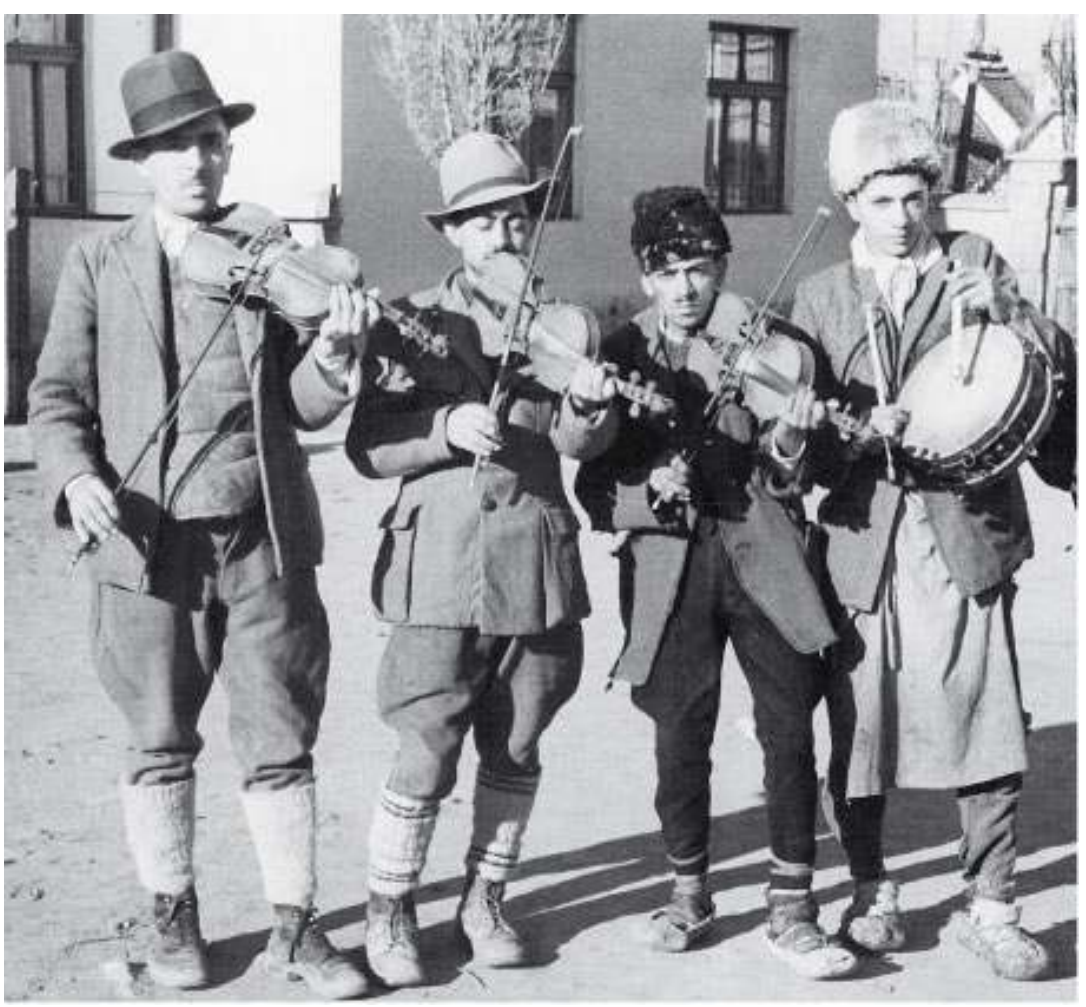

Photo : Arad, 1956 


\section{Une musique-miroir du présent}

9 Par rapport aux musiques traditionnelles encore vivantes en Roumanie, la musique « du Banat ", "serbe", "turque", "tsigane " ou "polluée " est excessive sous tous ses aspects. Elle étale des timbres mordants ou, au contraire, excessivement doux, mais toujours ostentatoires et, qui plus est, déformés par des sonorisations de qualité médiocre ; des rythmes plutôt inhabituels, que les gens identifient comme "orientaux " (le sont-ils vraiment?); des lignes mélodiques plates, répétitives, inexpressives; des accompagnements harmoniques construits de manière fantaisiste, mais d'un goût précaire ; des vers prosaïques, voire licencieux. Elle est cependant fermement ancrée dans le présent, dans la vie quotidienne des gens des villages et des villes. Les textes de ses chansons parlent obstinément de la vieille petite maman, abandonnée dans sa maison au fond du hameau par ses nombreux enfants émigrés en ville (près de la moitié des habitants des villes de Roumanie sont nés à la campagne, méprisent le village, mais ressentent une vive nostalgie à son égard); ils traitent aussi de « sponsors ", d'affaires, de tricheries, de bagnes, de procès et de pots-de-vin passés aux juges ; ou encore des idéaux de vie du Roumain ordinaire (fêtes, nourriture abondante, femme fidèle et respectable, amante volcanique, enfants et téléviseur en parfait état de marche); d'histoires d'amour aux relents de feuilletons sud-américains. En voici un exemple, parmi les plus communs :

\begin{tabular}{|l|l|}
\hline «Stinge, fă, telecomanda & «Coupe la télécommande \\
\hline \hline Că s-a termenat 'Cassandra’” & Car le film 'Cassandra' vient de finir \\
\hline Bine că s-a termenat & C'est bien qu'il soit fini \\
\hline Că mă lăsai nemâncat !» & Sinon tu me laisserais crever de faim ! \\
\hline
\end{tabular}

10 La musique est souvent parsemée de passages virtuoses improvisés, libres, spontanés et pleins d'énergie. Sous l'angle de la vitalité et de l'authenticité de l'expression, elle est tout le contraire de la musique folklorique ("décente", "conforme» à la perspective de l'esthétique officielle, feignant l'optimisme, rigide, menteuse et dépourvue de force réelle), qui poursuit sa destinée grise mais pérenne. Par sa vigueur, sa grossièreté et son exubérance, la nouvelle musique est en accord avec la vie des Roumains d'aujourd'hui : rien de plus naturel que son succès, même si ce dernier demeure plutôt superficiel, comme nous allons le voir. Les citadins cultivés la détestent de tout cœur et la trouvent ignoble. Leur animosité a des raisons tout d'abord esthétiques; mais elle est en outre motivée par un profond mépris pour la musique populaire en général, disqualifiée à cause de son chauvinisme et de ses longs compromis avec le pouvoir politique, et aussi par le fait qu'elle est jouée surtout par des Tsiganes, par l'aversion pour les faubourgs sordides et humiliants dans lesquels, en définitive, ils vivent également, etc. 


\section{Une musique globalisante}

11 La nouvelle musique a une identité ethnique confuse, ou plutôt multiple, qui ne peut être comprise que par référence à l'espace balkanique dans son ensemble. Selon les circonstances, son style peut faire pencher la balance vers celui de l'une ou de l'autre des musiques nées dans ce périmètre : la serbe, la turque, la roumaine, la macédonienne, etc. Souvent, elle emprunte massivement des traits prélevés aux musiques traditionnelles d'une région tout en conservant une certaine ambiguïté relative à ses origines. Pour toutes ces raisons, elle pourrait être définie par le syntagme « musique de métissage panbalkanique », que j'utiliserai pour la nommer dans les lignes qui suivent.

12 La musique de métissage pan-balkanique se présente sous deux formes voisines, aussi importantes l'une que l'autre: la première, interprétée «sur le vif » lors des fêtes de noces, et la seconde, «normalisée » (c'est à dire transformée en bien de consommation). Elle englobe souvent, dans des proportions variables, des éléments rythmiques et de timbre empruntés à la musique pop-rock. Si elle n'était pas créée et interprétée par des lă utar ignorant totalement l'écriture musicale, elle pourrait être considérée comme de la world music. D'ailleurs, les similitudes des deux musiques - similitudes qui s'étendent jusqu'à leur fonction de musique d'ambiance et leurs couleurs sonores nécessairement artificielles, électroniques - ne sont pas négligeables et mériteraient un jour un examen plus attentif.

Fig. 3 : Janiaka Nolov et ses musiciens au restaurant Jaristea.

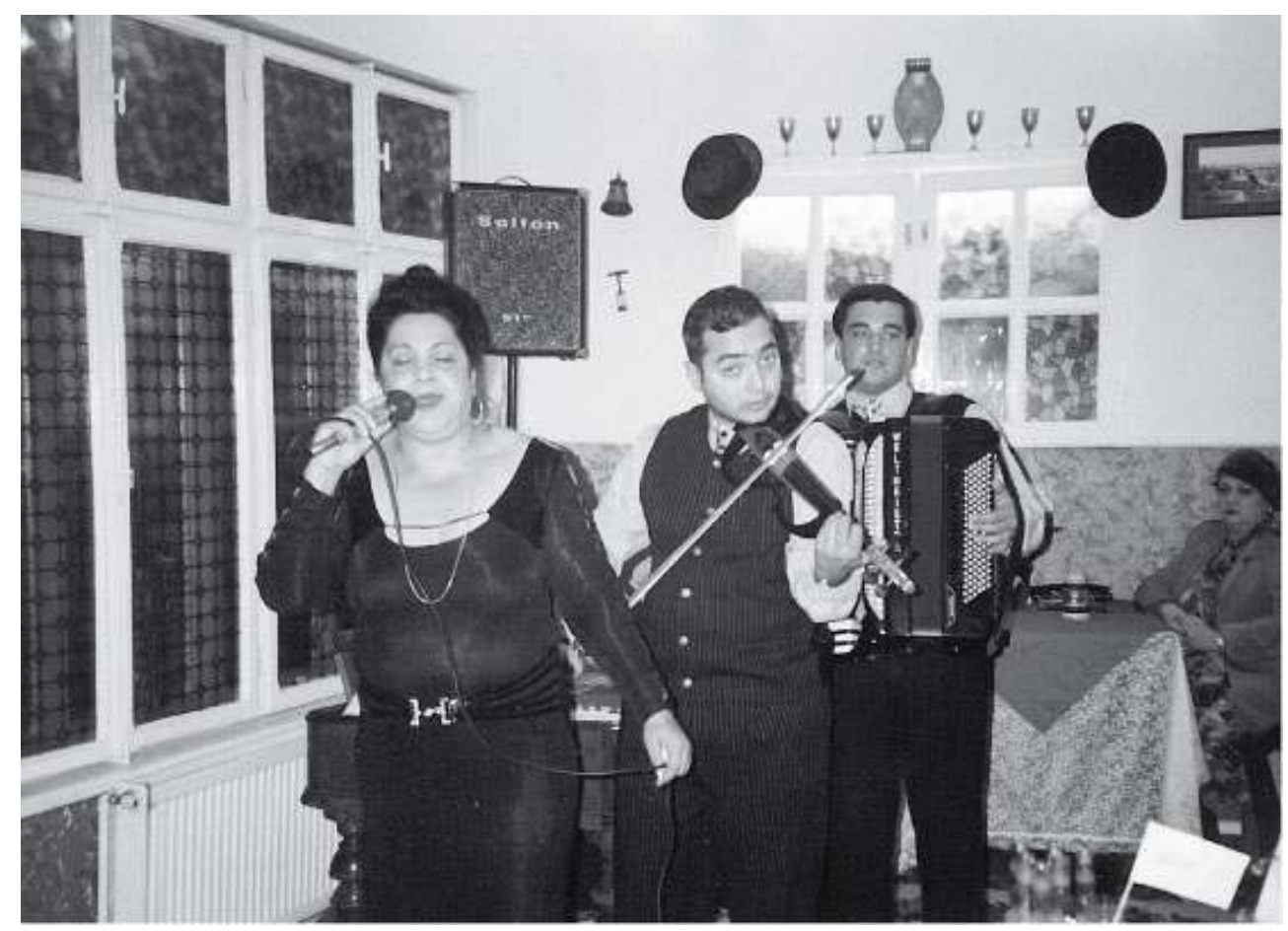

Photo : Valeriu Radulescu.

13 A ses débuts (les années 1970), la musique de métissage paraît encore procéder de l'effet de modernisation « naturelle » à laquelle s'expose toute musique de tradition orale. Plus tard, il devient de plus en plus clair que cette modernisation - nettement plus agressive 
que dans le passé - a engendré une musique différente, que ceux qui s'en servent reconnaissent comme distincte et pour laquelle ils cherchent un nom approprié. La musique de métissage voit le jour dans le contexte des fusions culturelles en cours partout dans le monde. Elle peut être considérée comme le produit régional, propre au périmètre sud-est-européen, de la globalisation musicale; une globalisation qui va étrangement à contre-courant de l'intolérance ethnique dont les Balkans sont le théâtre, y compris par des conflits ouverts et des guerres civiles fratricides. Cette globalisation se développe sur fond de réceptivité accrue à toutes les musiques populaires du monde, y compris celles des Balkans en ce qui concerne la culture populaire européenne.

Dans les pays ex-communistes, la musique de métissage pan-balkanique prend corps en opposition à la musique folklorique officielle, figée, prévisible, triomphaliste et sans éclat. Les chercheurs américains qui l'ont étudiée la considèrent à juste titre comme une forme de résistance populaire masquée à l'oppression communiste, un prélude symbolique aux changements politiques qui auront lieu par la suite (voir Slobin 1996). Elle s'oppose aussi aux musiques traditionnelles ethniques/régionales/locales, dont elle se nourrit pourtant, mais qu'elle tend à détrôner.

Comment cette musique est-elle apparue ? La question n'est pas banale, compte tenu du fait que la Roumanie et la Bulgarie ont vécu quelques dizaines d'années dans un isolement presque total. On pourrait y répondre par un livre consistant, ayant comme préambule les rapports entre les musiques balkaniques au cours des siècles, et surtout au $\mathrm{xx} \%$ siècle. Le label que je lui ai apposé est-il correct ${ }^{8}$ ? Je n'en suis pas sûre. Ne s'agirait-il pas plutôt simplement d'une "musique tsigane»? Il est vrai que les musiciens tsiganes ont contribué de manière décisive à sa création et à sa diffusion. Mais il est clair qu'ils ne l'ont pas modelée uniquement à partir de leurs goûts, sans tenir compte des besoins et des exigences des communautés majoritaires auxquelles elle était et demeure destinée en priorité. On ne peut en outre pas ignorer le fait que les Tsiganes se l'approprient jusqu'à la considérer pratiquement comme un étendard ethnique. Les ethnomusicologues voulant afficher une attitude politiquement correcte évitent de mettre les sources de cette musique en corrélation avec une ethnie déterminée (ce qui est sage); toutefois, ils laissent entendre, lorsqu'ils se réfèrent à son contexte, qu'elle serait tout de même tsigane : cette opinion demeure à mon avis discutable. Il n'est cependant pas exclu qu'elle s'avère justifiée dans un futur plus ou moins proche. Dans l'intervalle, je me permets de rester sur ma position, en considérant que cette nouvelle musique est aujourd'hui une musique de métissage pan-balkanique. Au moins deux raisons justifient ma prudence : la circonspection des Rom, musiciens ou non, qui hésitent à la revendiquer et/ou de s'identifier entièrement à elle, et l'empressement avec lequel les Roumains xénophobes qui la méprisent - l'attribuent aux Tsiganes.

\section{Une musique contestée}

16 Cette musique et son succès fulgurant déclenchent en Roumanie des réactions de révolte et de résistance, liées ou non à la doctrine nationale-communiste et au comportement culturel qui en découle.

Ses adversaires les plus farouches sont les gens impliqués dans la diffusion de la musique folklorique officielle : les chefs d'orchestres populaires, les réalisateurs de spectacles et d'émissions de radio et de télévision, les activistes culturels, les producteurs de disques 
des éditions d'État, la plupart des fonctionnaires du Ministère de la Culture, etc. : en d'autres mots, les ex-apparatchiks de la culture communiste. Pour ceux-ci, la musique de métissage pan-balkanique (qu'ils appellent «tsigane » pour la disqualifier de façon plus efficace) est impure et condamnable en tant que telle, parce qu'elle ruine la soi-disant "spécificité nationale » que tout brave Roumain se doit d'aimer et de cultiver avec une ardeur toute patriotique. Chaque fois que l'occasion se présente - et pour eux, elle est fréquente car ils dominent encore les médias -, ils se lancent dans de vigoureuses diatribes contre cette musique, ses auteurs et ses colporteurs.

Les interprètes médiocres de musique folklorique « officielle » y sont tout aussi opposés : pour arrondir leurs fins de mois, ils la joueraient et la chanteraient volontiers au cours des fêtes de mariage; mais, comme ils ne trouvent pas d'employeurs, ils se plaisent à jouer le rôle des artistes clairvoyants et responsables qui refusent de contribuer à la destruction de la "vraie musique roumaine». (Les vedettes se taisent: interpréter les deux types de musique - qui ne sont d'ailleurs pas incompatibles - satisfait en même temps leur aspiration à la « gloire » nationale et leur désir de bien gagner leur vie.)

La troisième catégorie de contestataires est constituée de gens âgés, paysans et citadins des faubourgs de partout, pour qui la musique de métissage demeure quelque chose de plus ou moins étranger, inconfortable et fatigant. Lors des fêtes communautaires auxquelles ils prennent part, ils demandent parfois avec insistance aux lăutar de jouer leur musique, «notre musique, la musique de chez nous».

Enfin, les contestations les plus dignes d'être prises en considération proviennent des musiciens traditionnels de valeur. Pour eux, l'exécution de la musique de métissage est dégradante et même nocive, parce qu'elle affecte la qualité de leur chant ou de leur jeu instrumental ; sans parler du fait que, depuis son apparition, ils se voient " injustement » concurrencés par des lăutar au talent dérisoire, mais sans scrupules, prêts à jouer n'importe quoi n'importe comment pour de l'argent. (Il n'y a pas de doute que la nouvelle musique, moins complexe et moins difficile a exécuter que "l'ancienne», ouvre largement la voie aux imposteurs.) Les lăutar réputés profitent souvent de leur prestige personnel pour imposer a leurs auditeurs, ne serait-ce qu'une partie de la "vraie» musique d'autrefois. Certains poussent la probité jusqu'à refuser des engagements dans les villages où ils seraient contraints de ne jouer que de la musique de métissage. Les autres se résignent en soupirant à jouer « ce qu'on leur commande ». D'autres encore ont trouvé une façon assez habile d'y échapper: ils jouent sérieusement la musique traditionnelle locale et se bornent à diffuser des cassettes de musique métissée. Leur tricherie est quelquefois acceptée car leurs employeurs et leurs invités, très exigeants en ce qui concerne l'exécution de la musique traditionnelle, sont beaucoup plus tolérants lorsqu'il s'agit de musique « actuelle».

\section{Une musique à double jeu}

21 La plupart des non-musiciens n'ont pas d'opinion claire et nette sur la nouvelle musique. Au premier abord, ils déclarent qu'ils l'aiment de tout cœur. Pourtant, il est hors de doute qu'ils y sont attachés parce qu'elle leur permet de s'affirmer modernes, urbains, ouverts - de vrais "winners", pour emprunter une expression américaine qui commence a se répandre en Roumanie. Ils l'écoutent distraitement, comme par obligation, ou la diffusent à tue-tête en fond sonore de leurs conversations. Cette musique paraît d'ailleurs inhiber toute réaction corporelle en eux. « Dès que le taragot se met à jouer, les gens cessent de 
danser », dit un musicien du Pays de l'Oach, un "spécialiste » de la musique de métissage. Par contre, quand les lăutar reprennent leurs violons, altos, cymbalums et contrebasses, les mêmes gens s'éveillent et réagissent avec une implication émotionnelle et physique immédiatement perceptible. Les "Fêtes de la Musique Paysanne ", organisées chaque été à Bucarest par le Musée du Paysan, le montrent de manière frappante. Les habitants de la capitale, en bonne partie des campagnards récemment immigrés, ont d'habitude honte d'afficher leur passion pour la musique rurale - musique reliée à un mode de vie fruste, primitif, déprécié, qui était le leur et qu'ils veulent oublier aussi vite que possible. Mais, si la source de diffusion de cette dernière est « honorable »-c'est le cas des fêtes du musée, télévisées et favorablement jugées par des voix prestigieuses - ils oublient gêne et pudeur et se laissent envahir par l'immense plaisir de l'écouter. Parfois, ils font irruption dans les espaces de la fête en fracassant les portes ou en piétinant l'herbe des pelouses et commencent à danser comme des possédés. N'empêche que, le jour suivant, ils retournent docilement à la consommation de la musique de métissage qui leur paraît, sinon plus sécurisante, du moins plus recommandable du point de vue social.

Mais, en dépit de la précarité de son succès, de l'inconfort qu'elle peut susciter ou des voix parfois bien sonores de ses opposants, la musique de métissage pan-balkanique poursuit son chemin « roumain » en se fortifiant et en changeant constamment d'aspect. Sa charge symbolique l'emporte sans conteste sur ses particularités intrinsèques, lesquelles se révèlent souvent décevantes.

\section{NOTES}

1. Le taragot est un aérophone en bois à anche simple, un peu plus grand et nettement plus sonore que la clarinette, dont le timbre se rapproche de celui de la zurna balkanique. Inventé par un Hongrois il y a environ un siècle, le taragot est utilisé depuis quelques décennies surtout par les musiciens paysans roumains du Banat.

2. Les lautar sont les musiciens professionnels ou semi-professionnels des milieux populaires ruraux et/ou urbains.

3. Le Banat est divisé en deux parties par la frontière: le «Banat roumain» et le «Banat serbe».

4. A cette époque, les cassettes sont encore plutôt rares.

5. Ses détracteurs - intellectuels et pseudo-intellectuels - continuent cependant de la considérer comme «polluée» et «bâtarde».

6. Dans chacun de ces pays, on peut noter la tendance à attribuer une origine étrangère à cette musique. En 1995, par exemple, un de ces «tubes» passait pour être serbe en Roumanie, et roumain en Bulgarie.

7. "Cassandra» est un feuilleton à succès récemment diffusé sur l'une des chaînes de télévision roumaines.

8. La seule confirmation m'en est venue de la part de l'ethnomusicologue finlandais Vessa Kurkela, au cours d'une conversation informelle (1996). 


\section{RÉSUMÉS}

Dans cet article, l'auteur jette un coup d'œil sur la « nouvelle » musique orale qui explose de nos jours dans les villes et les villages de Roumanie et de tous les Balkans. En dépit de sa violence et de sa vulgarité, qui scandalisent les classes cultivées, cette musique contredit ou « adoucit » les tensions se manifestant dans la région. Effet d'un métissage musical pan-balkanique, elle prend toutefois des couleurs légèrement différentes selon l'aire culturelle ou elle est véhiculée. Malgré la cote d'amour dont elle jouit dans les milieux populaires, ceux-ci hésitent pourtant à la considérer comme leur musique, préférant l'attribuer aux «autres». Ces «autres» sont cependant difficiles a identifier : ils sont soit serbes, soit turcs, soit tsiganes, soit bulgares, soit lautari (musiciens professionnels, en bonne partie tsiganes)... Les dénominations de cette musique changent aussi chaque année, au rythme de ses transformations. Depuis deux ans c'est-à-dire depuis la rédaction de cet article - elle est appelée «orientale ». Au fond, cette dernière appellation pourrait être la plus appropriée, dans la mesure où elle circonscrit cette musique du point de vue ethnique et géographique de la façon la plus tolérante.

\section{AUTEUR}

\section{SPERANȚA RǍDULESCU}

Sperant, a Răudulescu, née en 1949, après des études en composition musicale et un doctorat en musicologie générale, se consacre graduellement à l'ethnomusicologie. En tant que chercheur a l'Institut d'Ethnographie et de Folklore, puis au Musée du Paysan de Bucarest, elle effectue de nombreuses recherches de terrain en Roumanie. Elle est l'auteur de quelques livres et articles concernant la musique des ensembles professionnels (largement composés de Tsiganes), la chanson lyrique, la musique folklorique officielle, les nouvelles musiques de métissage des Balkans, etc., et publie (surtout en Occident) des disques de musique traditionnelle roumaine. Elle est également l'auteur de quelques essais littéraires et d'un film ethnomusicologique, ainsi qu'occasionnellement productrice de spectacles, par plaisir et par nécessité. 